\title{
Bearing Locating Algorithm of Target based on Radial Basis Function Neural Network
}

\author{
Wang Zihao*, Tian Jie \\ Key Laboratory of Military Communication, Engineering University of CAPF, Xi'an, China \\ wantzh@aliyun.com, 1295353164@qq.com
}

Keywords: radial basis function neural network; pyroelectric infrared sensor; bearing-location

\begin{abstract}
Aiming at the high locating error and the underutilization of redundancy bearing measurement in Pyroelectric Infrared Sensor (PIR) location system, a novel method of bearing location based on Radial Basis Function Neural Network (RBFNN) is presented. After illustrating the region partition model of PIR sensor node, we take advantage of the K-means clustering method and the gradient-descent method to train the neural network. By comparing different sizes of training samples, we select a neural network model with lower locating error, and we have made a comparison of RBFNN and the geometric algorithm. The result of simulation shows that the neural network model has $18 \%$ higher locating accuracy and the locating error is much less than the geometric algorithm when the target is near the boundary of the detecting area.
\end{abstract}

\section{Introduction}

With the popularity of PIR's application, researchers come to do researches on target location method based on Wireless Sensor Nerworks (WSNs) and PIR technology. Dr. Qi Hao et al first studied the feasibility of the use of PIR in motion detection. In [1], with the Field of View (FOV) of PIR sensor nodes partitioned into several sub-regions and encoded, the locaction of target is estimated through grid approximation. In [2], a target location estimation method is put forward using the angular bisectors of the FOV. In [3], Probabilistic Neural Network (PNN) is used for assureing which sub-region the target is in. Then the crossing localization based on neural network region partition (CLBNNRP) method is used for estimating the accurate location of the target, which will largely improve the locating accuracy .

By now, most of the target location methods based on PIR focus on geometry and grid approximation algorithms, by measuring the angular information of target through sensor nodes. Geometric Algorithm(GA) is simple and clear, but the bearing error and the distance between target and sensor node may largely lead to loss of locating accuracy with large locating error.

Thus, this paper put forward a bearing location algorithm based on RBFNN. Section 2 presents the sensing model of the detecting area and the codes of every sub-region. Section 3 makes use of the nonlinear mapping relationship between numerous position information of training samples and the angular measurement that PIR sensor nodes acquire to establish a neural network model of target location. Section 4 demonstrates and analyses simulation results. Section 5 concludes the paper and points out the further study.

\section{The Sensing Model and Corresponding Code of Detecting area}

From the top view, the sensing area of single PIR sensor node is shown as Fig.1 (a). The FOV of PIR sensor node is a $90^{\circ}$ fan-shape area, whose radius is $10 \mathrm{~m}$. The fan-shape area is segmented into 8 sub-regions denoted as $\mathbf{r}=\left\{\mathrm{r}_{1}, \mathrm{r}_{2} \ldots \mathrm{r}_{8}\right\}$. When a target appears in one of the 8 regions, the corresponding region will be trigger and $r_{i}=1$, if not $r_{i}=0$. 


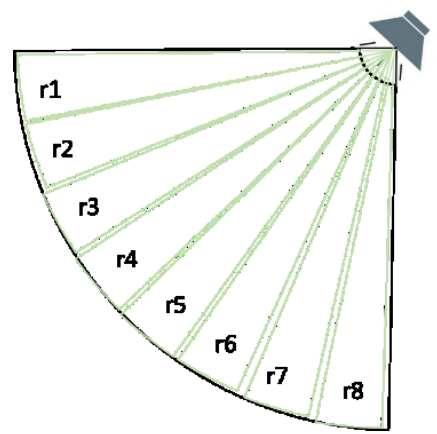

(a)

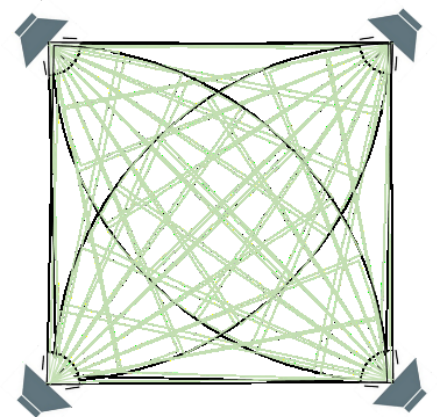

(b)

Fig.1. Detecting area of PIR sensor node

Table 1. Sensing area partition and the corresponding region code

\begin{tabular}{cccccc}
\hline Triggered region & $\begin{array}{c}\text { Binary } \\
\text { code }\end{array}$ & Decimal code & Triggered region & $\begin{array}{c}\text { Binary } \\
\text { code }\end{array}$ & $\begin{array}{c}\text { Decimal } \\
\text { code }\end{array}$ \\
\hline 1 & 1000 & 8 & 4,5 & 0011 & 3 \\
1,2 & 1100 & 12 & 5 & 0010 & 2 \\
2 & 0100 & 4 & 5,6 & 0111 & 7 \\
2,3 & 1110 & 14 & 7 & 1001 & 9 \\
3 & 1010 & 10 & 7,8 & 1111 & 15 \\
3,4 & 1011 & 11 & 8 & 0110 & 6 \\
4 & 0001 & 1 & None & 0000 & 0 \\
\hline
\end{tabular}

In general, considering that every target is not a mass point, every target is regarded as a circle whose diameter is $0.4 \mathrm{~m}$ in the $2 \mathrm{D}$ horizon space. When a target is near the boundary of two adjacent sub-regions, the two sub-regions will be triggered simultaneously resulting $r_{i}=1$ as well as $r_{i+1}=1$. When a target triggers only one sub-region, the angle bisector of the sub-region will be taken as the bearing measurement of the target. When a target triggers two adjacent sub-regions, the angle bisector of the two sub-regions will be taken as the bearing measurement of the target. According to [4], in the mentioned-above pattern, when the distance between target and sensor node is greater than $0.8 \mathrm{~m}$, a target will triggered two adjacent sub-region at most. Considering that the sensing radius of PIR sensor node is $10 \mathrm{~m}$, this pattern is applicable in most cases. Taking accuracy and precision into consideration, according to the dectecting area partition and the code design in [5], the binary code and the decimal code of sub-region are shown as Table 1.

\section{Target Location Method based on RBF Neural Network}

In the tranditional geometric algorithm, the angle measurement is not fully utilized while estimating the position of target. It usually uses two of the four angle measurement respectively and calculates the average value of results to estimate the target location, which leads to redundancy in the target position information and low locating accuracy .

This paper brings in the RBFNN for the bearing-location problem. PIR sensor nodes detect target in the sensing area and take the decimal code from 4 PIR sensor nodes into the RBFNN as input. Through a serial of calculation, the neural network will export the target's coordinate, utilizing the great generalization ability, fast learning convergence speed and strong nonlinear mapping ability of RBFNN. The traing algorithm of the RBFNN is explained in section 3. Through numerous training samples's study, the RBFNN will closely approach the nonlinear relationship between the coded sequence from PIR sensor nodes and the target position coordinate, which is supposed to reduce the effect of bearing error to target location. 4 PIR sensor nodes detect the situation of the 
sensing area, and the cooresponding coded squence will be put into RBFNN and the location of target will be estimated.

\section{A. The Target Location Model Based on RBFNN}

As is shown in Fig.1 (b), the detecting area is segmented into many irregular polygons. There is some nonlinear mapping relationship between the coded sequence of 4 PIR sensor nodes and the target position coordinate. It can be denoted as $f(R 1, R 2, R 3, R 4) \rightarrow \hat{T}(\hat{x}, \hat{y})$, as RBFNN can approach any nonlinear function. According to Cover theorem, the situation that the data can't be divided in low dimensional space will be more likely be solved in high dimensional space. Thus, it's available to establish the mapping model of the coded sequences from PIR sensor nodes to the target's position coordinate as $f(R 1, R 2, R 3, R 4) \rightarrow \widehat{T}(\hat{x}, \hat{y})$, from which the location of target can be estimated.

In this paper, we take the RBF as the mapping model. The Gaussian function is selected as the hidden layer activation function of RBFNN as the following equation

$$
\varphi_{i}(\mathrm{x})=\exp \left(-\frac{|| X-C_{i}||}{2 \sigma^{2}}\right)
$$

In Eq. $1, X=\left\{\left(R_{1 t}, R_{2 t}, R_{3 t}, R_{4 t}\right) \mid t=1,2, \ldots, q\right\}$ is denoted as the input vector, in which $R_{1 t}, R_{2 t}, R_{3 t}$, $R_{4 t}$ indicate that the $1^{\text {st }}, 2^{\text {nd }}, 3^{\text {rd }}, 4^{\text {th }}$ PIR sensor node's exporting coded sequence at time $t$. $C_{i}$ is denoted as the center of the $i^{\text {th }}$ radial basis function with the same dimension as $X . \sigma$ is denoted as the width of radial basis function around the center, while $m$ represents the number of the neurons in hidden layer. $\| X-C_{i}||$ is denoted as the Euclid norm of $X-C_{i}$, which represents the distance between the input and the center of the radial basis function. The activation level of neuron will be higher when the distance between the input and the center is closer, consequently the output of $\varphi_{i}(x)$ will be larger. Conversely, the output of $\varphi_{i}(x)$ will be smaller.

In the radial basis function, the mapping relationship between hidden layer and the output layer is linear, and the target's position coordinate can be denote as the linear weighted sum of the output of each neuron in the hidden layer, as is shown in Eq.2.

$$
\widehat{\mathrm{T}}(\hat{\mathrm{x}}, \hat{\mathrm{y}})=\sum_{i=1}^{m} \omega_{i} \varphi_{i}(|| \mathrm{X}-\mathrm{Ci}||)
$$

B. Training Algorithm

Generally, there are three parameters that need to be studied in RBFNN, such as the center of radial basis function $C_{i}$, the width of radial basis function around the center $\sigma_{i}$ and the weight between the hidden layer and the output layer $\omega_{i}$. In this paper, we choose the unsupervised learning algorithm to get the centers of radial basis function, then we trained the three parameters by gradient descent method to optimize. The training steps is as follows

(1) Generate training samples and preprocess: The coordinates of 4 PIR sensor nodes are $(0,0)$, $(10,0),(10,10),(0,10)$. In the detecting area of PIR sensor node, targets' position are randomly generated in range of $[0,10]$. By coded sequences' program, the cooresponding decimal coded sequences are generated as $X_{j}\left(R_{1}, R_{2}, R_{3}, R_{4}\right)$.

(2) The centers of the radial basis function are calculated by K-means clustering algorithm. And the $\sigma_{i}$ and the $\omega_{i}$ are randomly initialized.

(3) The three parameters, $C_{i}$, $\sigma_{i}$ and $\omega_{i}$, are studied and optimized by gredient descent method

i. The cost function is shown as Eq.3.

$$
\mathrm{E}=\frac{1}{2} \sum_{j=1}^{N} e_{j}^{2}
$$


where $e_{j}$ is denoted as the error of the $\mathrm{j}^{\text {th }}$ sample, and $d_{j}$ represents the expected output. And $e_{j}$ can be calculated as Eq.4.

$$
e_{j}=d_{j}-f\left(X_{j}\right)=d_{j}-\sum_{i=1}^{m} \omega_{i} \varphi_{i}\left(|| X_{j}-C_{i}||\right)
$$

ii. In order to minimize the objective function, the correction of each parameter is proportional to the gradient. After each iteration, the parameters will be regulated at a speed of $\eta=0.001$ to the negative direction of the error gradient. While iteration reaches 1000 or the drop height of gradient is less than 0.001 , the gradient descent will stop. The regulation of the 3 parameters is shown as following

$$
\begin{aligned}
& \Delta c_{i}=-\eta \frac{\partial E}{\partial c_{i}}=\eta \frac{\omega_{i}}{\sigma_{i}^{2}} \sum_{i=1}^{m} e_{j} \varphi_{i}\left(\left|X_{j}-C_{i}\right| \mid\right)\left(X_{j}-C_{i}\right) \\
& \Delta \sigma_{i}=-\eta \frac{\partial E}{\partial \sigma_{i}}=\eta \frac{\omega_{i}}{\sigma_{i}^{3}} \sum_{i=1}^{m} e_{j} \varphi_{i}\left(|| X_{j}-C_{i}||\right)|| X_{j}-C_{i} \|^{2} \\
& \Delta \omega_{i}=-\eta \frac{\partial E}{\partial \omega_{i}}=\eta \sum_{i=1}^{m} e_{j} \varphi_{i}\left(\left|X_{j}-C_{i}\right| \mid\right)
\end{aligned}
$$

It is the training process of $\mathrm{N}$ training samples, after which the RBFNN model is capable of estimating the target's position coordinate in a $10 \mathrm{~m} * 10 \mathrm{~m}$ detecting area.

\section{Simulations}

In order to verify the effectiveness of the RBFNN model, a large number of simulations are carried out to compare the RBFNN model with the GA.The simulation scene is shown in Fig.1(b). Four PIR sensor nodes' coordinates are $(0,0),(10,0),(10,10),(0,10)$. We use the RBFNN model and the GA to estimate the same target in detecting area.

\section{A. Effect of Training Sample Size on Target Locating accuracy}

The size of training samples has a great influence on the locating accuracy while using the RBFNN model. So it is significant to research on the size of training samples.

First, we randomly generated 3000 training samples as mentioned above. Second, we took the first 100, 200, 300...3000 targets' information as input of the RBFNN model, and the models we trained were named RBF100, RBF200, RBF300...RBF3000. Third, we randomly generated 100 points as test samples, whose position coordinates were estimated by the RBFNN models.

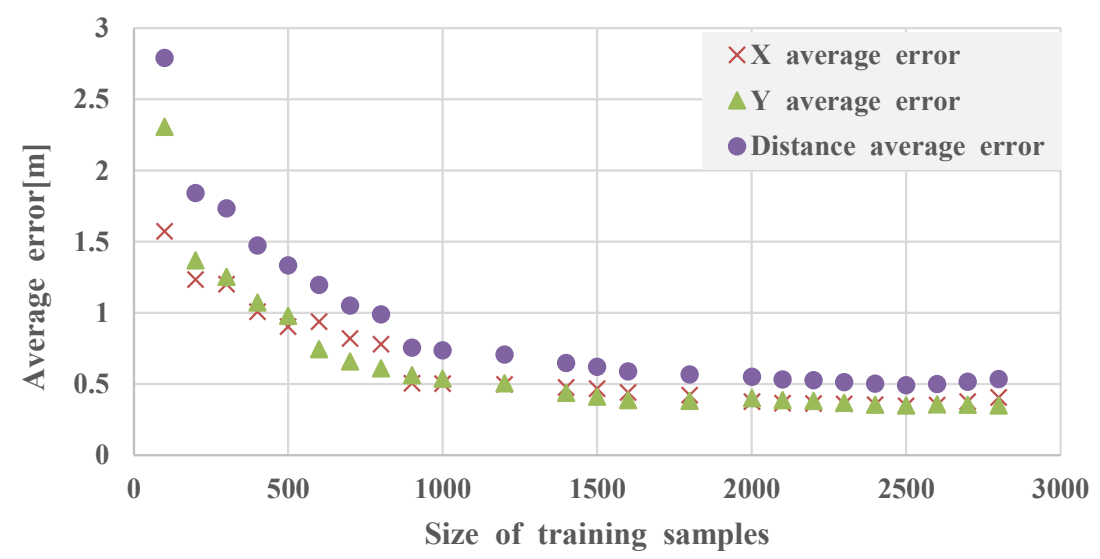

Fig. 2. Effect of sample size on locating accuracy 
As is shown in Fig. 2, when the number of samples increases from 100 to 1000, the average locating error decreases rapidly from $2.8 \mathrm{~m}$ to about $0.75 \mathrm{~m}$. When the number of training samples increases from 1000 to 2500 , the average locating error decreases slowly and reaches $0.5 \mathrm{~m}$ while the number of training samples is 2500 . When the number of training samples increases from 2500 to 2800 , the average locating error increases slowly. According to the simulation result above, the RBF2500 is selected to be the best RBFNN model to next step simulation.

B. Target Locating Simulation

(1) Simulation 1: target's initial position is $(4,0.5), v_{x}=0 \mathrm{~m} / \mathrm{s}, v_{y}=0.5 \mathrm{~m} / \mathrm{s}$. RBFNN and GA estimate the target's location every 1 second. The location result is shown in Fig. 3 (a).

(2) Simulation 2: target's initial position is $(2.4,0.67), v_{x}=0.4 \mathrm{~m} / \mathrm{s}$, and the path is $y=$ $\left(5^{*} \mathrm{x} / 3\right)-10 / 3$. RBFNN and GA estimate the target's location every 1 second. The location result is shown in Fig. 3 (b).

(3) Simulation 3: target's initial posit is $(1.5,5.2), v_{x}=0.35 \mathrm{~m} / \mathrm{s}$, and the path is $y=\sin (\mathrm{x})+2+\operatorname{sqrt}(\mathrm{x})+\operatorname{sqrt}(\operatorname{abs}(\sin (\mathrm{x})))$. RBFNN and GA estimate the target's location every 1 second. The location result is shown in Fig. 3 (c).

In the three simulations, the paths of the target are vertical, diagonal and curve respectively, which are representative. According to Fig.3, in the three simulation, the continuous RBFNN location is more close to the trajectory of the target.

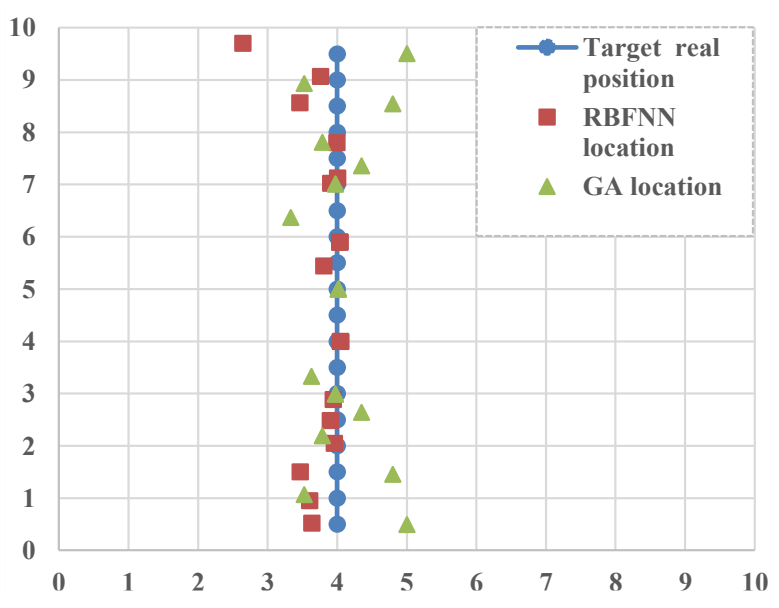

(a) Simulation 1

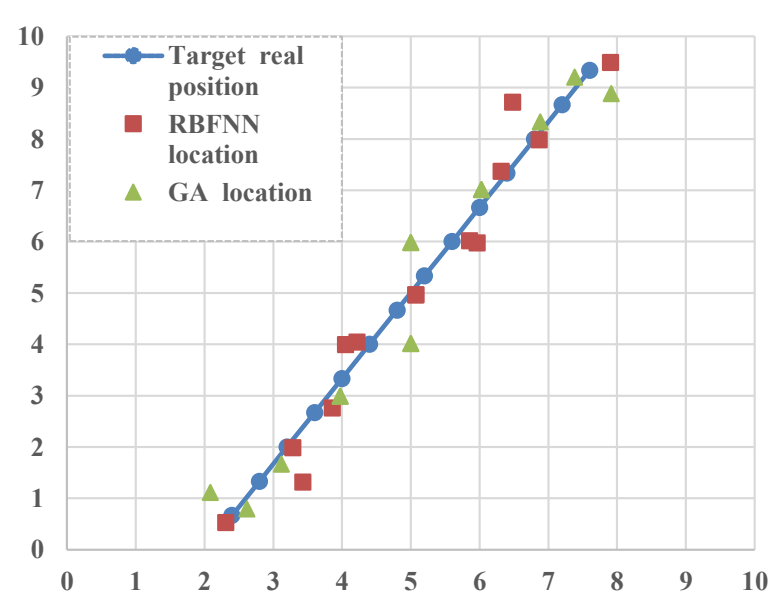

(b) Simulation 2

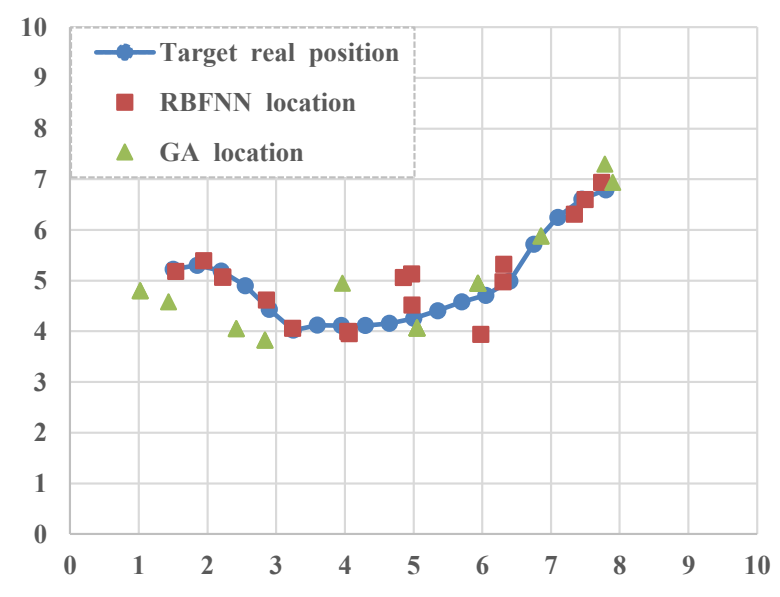

(c) Simulation 3

Fig. 3. Simulations using RBFNN and GA 
Table 2. Locating error of two algorithms

\begin{tabular}{|c|c|c|c|c|c|}
\hline \multirow{2}{*}{ Locating method } & \multirow{2}{*}{ Simulation } & \multicolumn{3}{|c|}{ Average error [m] } & \multirow{2}{*}{$\begin{array}{c}\text { Accuracy } \\
\text { improvement [\%] }\end{array}$} \\
\hline & & $X$ & $Y$ & Distance & \\
\hline GA & \multirow{2}{*}{1} & 0.36 & 0.21 & 0.50 & \multirow{2}{*}{18.06} \\
\hline RBFNN & & 0.22 & 0.24 & 0.41 & \\
\hline GA & \multirow{2}{*}{2} & 0.32 & 0.29 & 0.49 & \multirow{2}{*}{23.31} \\
\hline RBFNN & & 0.27 & 0.18 & 0.38 & \\
\hline GA & \multirow{2}{*}{3} & 0.37 & 0.36 & 0.57 & \multirow{2}{*}{40.05} \\
\hline RBFNN & & 0.25 & 0.20 & 0.34 & \\
\hline
\end{tabular}

As is shown in Table 2, in simulation 1, the average distance error of RBFNN and GA are $0.41 \mathrm{~m}$ and $0.50 \mathrm{~m}$ respectively. The average locating accuracy in distance increases by $18.06 \%$. In simulation 2, the average distance error of RBFNN and GA are $0.38 \mathrm{~m}$ and $0.49 \mathrm{~m}$ respectively. The average locating accuracy in distance increases by $23.31 \%$. In simulation 3, the average distance error of RBFNN and GA are $0.34 \mathrm{~m}$ and $0.57 \mathrm{~m}$ respectively. The average locating accuracy in distance increases by $40.05 \%$. The locating performance of RBFNN model is superior to the GA in most cases, which proves RBFNN's effectiveness.

RBFNN has taken good advantage of all the bearing measurement, whose great generalization ability and strong nonlinear mapping ability contribute to the improvement on locating accuracy. Meanwhile, its root-mean-square error (RMSE) increased a little, but the influence was insignificant comparing to its improvement in locating accuracy.

\section{CONCLUSION}

In this paper, a target locating method is proposed based on region partition with coded sequence and RBFNN for PIR sensor nodes. The detecting area is segmented into 8 sub-regions and encoded uniformly. PIR sensor nodes will be activated if a target appears in sub-regions and output a coded sequence about the target based on bearing measurement. RBFNN takes the coded sequence as input and estimates the position of target, which is trained by thousands of samples beforehand. Comparing with the GA, the simulation results have demonstrated that the algorithm of this paper based on RBFNN has significantly improved the locating accuracy without additional information.

\section{References}

[1] Q. Hao, D. J. Brady, B. D. Guenther, J. B. Burchett, M. Shankar and S. Feller, Human Tracking With Wireless Distributed Pyroelectric Sensors, IEEE Sensors Journal, 6.6(2006):1683-1696.

[2] Bo Yang, Jing Luo, Qi Liu, A novel low-cost and small-size human tracking system with pyroelectric infrared sensor mesh network, Infrared Physics \& Technology, 63.2(2014):147-156.

[3] Bo Yang, Xiaoshan Li, Jing Luo, A novel multi-human location method for distributed binary pyroelectric infrared sensor tracking system: Region partition using PNN and bearing-crossing location, Infrared Physics \& Technology, 68(2014):35-43.

[4] M. Shankar, J. B. Burchett, Q. Hao, B. D. Guenther, \& D. J. Brady. Human-tracking systems using pyroelectric infrared detectors, Optical Engineering, 45.10(2006):106401.

[5] Y. Yuebin, F. Guodong, G. Xuemei and W. Guoli, Compressed infrared bearing sensor for human localization: Design and implementation, International Conference on Information and Automation (ICIA), Shenyang, 2012:936-940. 